\title{
Analysis of Heart Rate Variability Influence on Heart Rate Turbulence Using Boosted Regression Trees in Heart Failure Patients
}

\author{
O Barquero-Pérez ${ }^{1}, \mathrm{~S}_{\text {Cantero }}{ }^{1}, \mathrm{R}_{\text {Goya-Esteban }}{ }^{1}, \mathrm{C}_{\text {Figuera-Pozuelo }}{ }^{1}$, \\ A García-Alberola ${ }^{3}$, JL Rojo-Álvarez $^{1,2}$ \\ ${ }^{1}$ Department of Signal Theory and Communications, University Rey Juan Carlos, Madrid, Spain \\ ${ }^{2}$ Center for Computational Simulation, Polytechnic University of Madrid, Spain \\ ${ }^{3}$ Unit of Arrhythmias, Hospital Universitario Virgen de la Arrixaca, Murcia, Spain
}

\begin{abstract}
Heart Rate Turbulence (HRT) is a physiological phenomenon used as cardiac risk stratification criterion. The relationship between Heart Rate Variability $(H R V)$ and HRT has been documented in the literature. However, the influence of HRV on HRT using individual tachograms has not been addressed. Our aim was to propose a nonparametric model, based on Boosted Regression Trees (BRT), of turbulence slope (TS) as a function of coupling interval (CI), Age, Sex, and HRV time-domain indices. We used data sets of myocardial infarction (MI) and heart failure (HF) patients. HRV was assessed on 3-min NN interval segments just before to individual ventricular premature complex (VPC) tachograms. We proposed to model TS as a function HRV indices using BRT, which is an ensemble approach to build regression models using several small trees. We segmented data into high risk and low risk according to HRT cut-off values of TS. Variables related to $H R V$ were the most important explaining the HRT in low risk patients, while in patients with high risk, $C I$ and heart rate just before the VPC played an important role explaining the HRT response.
\end{abstract}

\section{Introduction}

Heart Rate Turbulence (HRT) is the physiological response to a spontaneous ventricular premature complex (VPC). In normal subjects, it consists of an initial acceleration and subsequent deceleration of the sinus heart rate. Heart rate variability (HRV) reflects the regulation of the heart rate by the autonomic nervous system (ANS). Both, HRT and HRV, have been shown to be strong risk stratification predictors in patients with high-risk of cardiac disease [1-3].

It has been documented in the literature the influence of several physiological factors on the HRT [2]. The heart rate affects the strength of the HRT response, in a way that
HRT is reduced at high heart rate. VPC prematurity also influences the HRT response. So, in agreement with the baroreflex source of HRT, the more premature the VPC, the stronger the HRT response should be [4,5]. It has been studied in the literature some interaction effect between sex and age on HRT [6,7]. Finally, there are evidences of correlation between HRT and HRV on 24-hour Holters, since both are under the influence of the ANS [8].

In this work, we propose to use a nonparametric model of the HRT response for each individual VPC as a function of different factors, namely, the previous heart rate just seconds before the VPC (sinus cardiac length, $S C L$ ), the VPC prematurity (coupling interval, $C I$ ), sex, age and HRV time-domain indices, computed on 3-min NN interval segments before the VPC. We propose to model this relationship using boosted regression trees (BRT). The aim is to model the effect of the HRV on the HRT using 24-hour Holter from myocardial infarction (MI) and heart failure (HF) patients.

The structure of the paper is as follows. In Section 2, HRT and HRV assessment is detailed. In Section 3, BRT model is explained. In Section 4, data sets are detailed. In Section 5, results are reported. Finally, in Section 6, conclusions are presented.

\section{Heart rate turbulence and heart rate variability}

HRT is usually assessed by two parameters, Turbulence Onset $\left(T_{O}\right)$ and Turbulence Slope $\left(T_{S}\right)$. Both parameters are usually computed on an averaged VPC tachogram built using all available individual VPC tachograms from 24hour Holters [2]; even though there exist some other approaches to quantify HRT $[9,10]$. TO assesses the amount of sinus acceleration following a VPC, and it is defined as the percentage difference between the heart rate immediately following the VPC and the heart rate immediately preceding the VPC. TS represents the rate of sinus decel- 


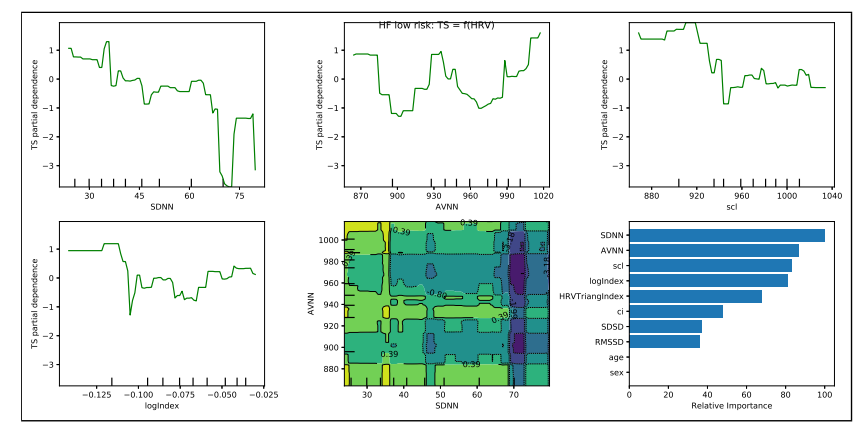

(a)

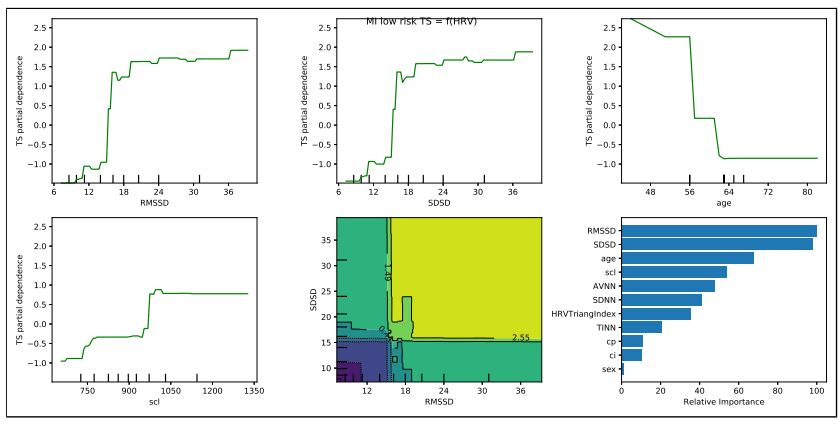

(c)

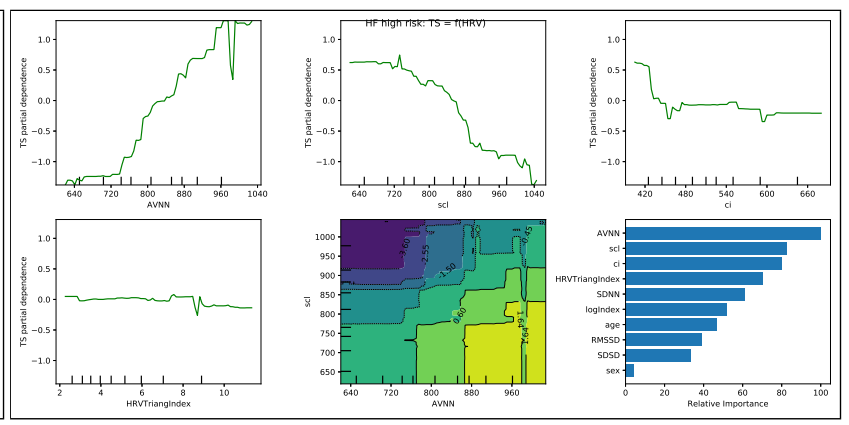

(b)
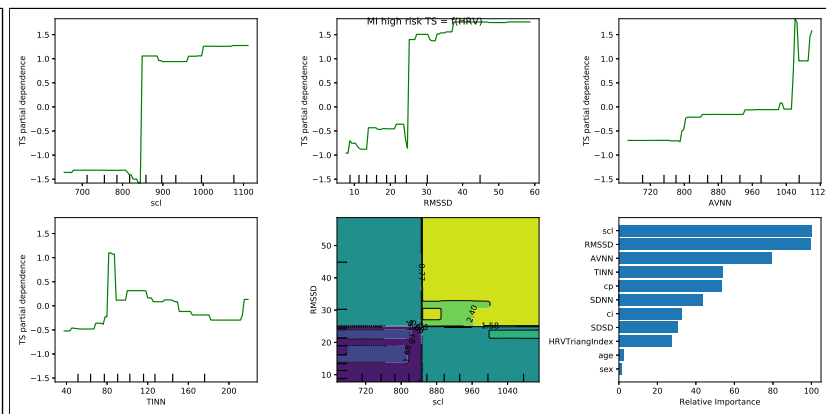

(d)

Figure 1. Summary of results, PDP (one-way and two-way) and feature relative importance of modeling HRT using BRT for HF low risk (a), HF high risk (b), MI low risk (c), and MI high risk (d).

eration that follows sinus acceleration, and it is defined as the maximum positive regression slope assessed over any 5 consecutive sinus rhythm RR-intervals within the first 15 sinus rhythm RR-intervals after the VPC [2].

HRV is usually assessed by time-domain indices (statistical and geometrical), which are computed on NN-interval time series from 24-hour Holter recordings. In this work, HRV time-domain indices are computing on 3-min segments before each individual VPC tachogram, only segments with more than $90 \%$ of NN intervals (sinus beats) were allowed. The aim is to assess the status of the ANS just before the VPC. HRV is usually assessed on 5-min segments, however this would lead to very few valid VPC tachograms. The following statistical time-domain indices were calculated: $A V N N$, average of all $\mathrm{NN}$ intervals; $S D N N$, standard deviation of all NN intervals; $r M S S D$, square root of the mean of the sqaures of differences between adjacent $\mathrm{NN}$ intervals; $S D S D$ standard deviation of differences between adjacent NN intervals. Also, the following geometrical time-domain indices were calculated: Triangular index, $H R V_{\triangle}$, total number of all NN intervals divided by the height of the histogram of all NN intervals; TINN, baseline width of the minimum square difference triangular interpolation of the highest peak of the histogram of all $\mathrm{NN}$ intervals.

\section{Boosted tree regression model}

We propose to model the HRT, as assessed by a parameter $T_{s}$, as a function of the following explanatory variables: $S_{c l}$ (sinus cardiac length just before de VPC), $C_{i}$ (coupling interval), $C_{p}$ (compensatory pause after the VPC), $A$ (age), $S$ (sex), and HRV time-domain indices, namely $A_{v n n} S_{d n n}, r_{m s s d}, S_{d s d}, H R V_{\triangle}, T_{i n n}$ :

$$
\begin{aligned}
T= & f\left(S_{c l}, C_{i}, C_{p}, A, S, A_{v n n} S_{d n n},\right. \\
& \left.r_{m s s d}, S_{d s d}, H R V_{\triangle}, T_{i n n}\right)
\end{aligned}
$$

Function $f$ is learnt using BRT, which is a regression method that adaptively combines large number of, relatively simple, tree models [11]. This method has been widely used to generate predictive models in ecological and biological studies [12]. The BRT estimation, $\hat{f}(x)$, is obtained sequentially as follows

1. Set $\hat{f}(x)=0$ and $r_{n}=T_{S, n}$ for all the $n$ VPC tachograms available, where $r_{n}$ are the residuals.

2. For $b=1,2, \ldots, B$, repeat

(a) Fit a small tree, $\hat{f}^{b}$ to the training data $\left\{x_{n}, r_{n}\right\}$, where explicative variables are in vector $x_{n}$ and response variable is $r_{n}$.

(b) Update $\hat{f}$ by adding a shrunken version of the new small tree $\hat{f}^{b}$ :

$$
\hat{f} \leftarrow \hat{f}+\lambda \hat{f}^{b}
$$


(c) Update the residuals, $r_{n}$,

$$
r_{n} \leftarrow r_{n}-\lambda \hat{f}^{b}
$$

3. Finally, output the $B R T$ model:

$$
\hat{f}=\sum_{b=1}^{B} \lambda \hat{f}^{b}
$$

The main idea behind BRT is to learn slowly. A new small tree (with few terminal nodes) is fit using the current residuals and then added to $\hat{f}$, so that $\hat{f}$ is slowly improved in areas where it does not perform well previously [13].

BRT has three tuning parameters, namely, the number of trees $B$, the shrinkage parameter $\lambda$ that controls the rate at which BRT learns, and the number of splits in each tree, which controls the complexity of the boosted ensemble, it also controls the interaction order between explanatory variables in the model. Parameters were tuned using 10fold cross-validation, which is a usual procedure [13].

Unlike simple regression trees, BRT models can be more difficult to interpret. However, they can provide with some summary statistics that allow a better understanding of the final model and assess the feature importances. The relative importance and the partial dependence plots, PDP, are two of such statistics. The relative importance measures the contribution of each explanatory variable to the final model [11]. PDP are graphical tools to quantify the effect of one variable on the response after accounting for the average effects of the remaining variables in the model.

\section{Data sets}

We compared the proposed approach on two data sets, one with HF patients and another with MI patients. HF data set consists of 42-hour Holter recordings from 66 decompensated HF patients ( $65 \pm 14$ years, 15 women) with a left ventricular ejection fraction lower or equal than $35 \%$, collected during an observational multicenter study (VPredict+) comprising six different spanish hospitals, namely, Hospital Universitario Virgen de la Arrixaca (Murcia), Hospital Universitario La Paz (Madrid), Hospital Universitario de Madrid Montepríncipe and Sanchinarro, Hospital Universitario Virgen de las Nieves (Granada), and Hospital Clinico Universitario (Valencia). The MI data set consists of 24-hour Holter recordings from 61 post-myocardial infarction patients (64 \pm 9 years, 18 women) who underwent emergency coronary angiography, and, when appropriate, percutaneous infarction revascularization at University Hospital Virgen de la Arrixaca [14].

Both data sets were split into two different subsets, namely, a low risk subset, which comprised with $T_{S} \geq 2.5$ $\mathrm{ms} / \mathrm{RR}$ and $T_{O} \leq 0$, and a high risk subset with $T_{S}<2.5$ $\mathrm{ms} / \mathrm{RR}$ and TO $>0 \%$. These $T_{S}$ and $T_{O}$ cutoff values are commonly used in most clinical studies, where $T_{S}>2.5$ $\mathrm{ms} / \mathrm{RR}$ and $T_{O}<0 \%$ are considered as normal, and they were proposed using data from different post-infarction studies [2].

\section{Results}

Figure 1 shows one-way, two-way PDP and feature relative importance for HF low risk 1(a), HR high risk 1(b), MI low risk 1(c), and MI high risk 1(d) using BRT.

The three most important features for HF low risk were $S_{d n n}, A_{v n n}$, and $S_{C} L$. There were a negative relationship between SDNN and TS and SCL and TS. The three most important features for $\mathrm{HF}$ high risk were AVNN, scl and ci. There existed a positive relationship between AVNN and Ts and a negative relationship between TS and scl. Conditions just near VPC (i.e. SCL and CI) had more influence on HRT response for high risk than for low risk patients.

The three most important features for MI low risk patients were $r_{m s s d}, s_{d s d}$, and $a g e$, whereas for MI high risk were $S_{c l}, r_{m s s d}$ (very close), and $A_{v n n}$. Interestingly, there seemed to be a two-state behaviour, that can be observed in the relationship betwen $r_{M S S D}$ and $T_{S}$ and $S_{C L}$ and $T_{S}$. There is a value for $r_{m s s d}$, and $S_{c l}$, from which the value of the $T_{S}$ parameter changes from small to high values. The relationship between $T S$ and the two most important variables seems to be smoother on HF patients. For both data sets, conditions just near VPC had more influence on high risk patientes, than the general ANS status.

\section{Conclusions}

In this work we propose to use BRT to model the relationship between HRT parameter $T S$ and variables $S C L$, $C I$, Age, and Sex and six HRV time domain indices, both statistical and geometrical. HRV was assessed on 3-min NN interval segments just before every VPC. The model was fitted using data from MI and HF decompensated patients. Datasets were split into two different groups, namely, low risk and high risk groups according to $T_{S}$ and $T_{O}$ cut-off values reported in the literatue.

Results suggested that ANS status, assessed by HRV on the 3 previous minutes to the VPC, have higher influence on HRT response on low risk patients. In high risk patients, the heart rate just before the VPC has more influence on the HRT response. Interestingly HF patients showed a negative relationship between $S C L$ and TS, which is a the opposite behavior to that reported in the literature. For both data sets, conditions just near VPC had more influence on high risk patients, than the general ANS status.

Further work should be directed to incorporate HRV frequency domain indices, which allows to better characterize the ANS. Also, comparing HRT dynamics, regarding the proposed explanatory variables, between different pa- 
tient groups may give some insight on cardiovascular risk stratification.

\section{Acknowledgements}

This work has been partially supported by Research Projects from the Spanish Government TEC2016-75361R, TEC2013-46067-R and TEC2016-75161-C2-1-R, and TEC2016-81900-REDT.

\section{References}

[1] Schmidt G, Malik M, Barthel P, Schneider R, Ulm K, Rolnitzky L, Camm AJ, Bigger JT, Schömig A. Heart-rate turbulence after ventricular premature beats as a predictor of mortality after acute myocardial infarction. Lancet 1999; 353(9162):1390-1396.

[2] Bauer A, Malik M, Schmidt G, Barthel P, Bonnemeier H, Cygankiewicz I, Guzik P, Lombardi F, Müller A, Oto A, Schneider R, Watanabe M, Wichterle D, Zareba W. Heart rate turbulence: standards of measurement, physiological interpretation, and clinical use: International Society for Holter and Noninvasive Electrophysiology Consensus. Journal of the American College of Cardiology 2008; 52(17):1353-1365.

[3] Camm AJ, Malik M, Bigger J, Breithardt G, Cerutti S, Cohen RJ, Coumel P, Fallen EL, Kennedy HL, Kleiger RE, et al. Heart rate variability: standards of measurement, physiological interpretation and clinical use. task force of the european society of cardiology and the north american society of pacing and electrophysiology. Circulation 1996; 93(5):1043-1065.

[4] Schwab JO, Shlevkov N, Grunwald K, Schrickel JW, Yang A, Lickfett L, Lewalter T, Lüderitz B. Influence of the point of origin on heart rate turbulence after stimulated ventricular and atrial premature beats. Basic research in Cardiology 2004;99(1):56-60.

[5] Barquero-Pérez Ó, Figuera C, Goya-Esteban R, MoraJiménez I, Gimeno-Blanes FJ, Laguna P, Martínez JP, Gil E, Sörnmo L, García-Alberola A, et al. On the influence of heart rate and coupling interval prematurity on heart rate turbulence. IEEE Transactions on Biomedical Engineering 2017;64(2):302-309.

[6] Schwab J, Eichner G, Veit G, Schmitt H, Lewalter T, Lüderitz B. Influence of basic heart rate and sex on heart rate turbulence in healthy subjects. Pacing and clinical electrophysiology 2004;27(12):1625-1631.

[7] Schwab J, Eichner G, Shlevkov N, Schrickel J, Yang A, Balta O, Lewalter T, Lüderitz B. Impact of age and basic heart rate on heart rate turbulence in healthy persons. Pacing and Clinical Electrophysiology 2005;28:S198-S201.

[8] Cygankiewicz I, Wranicz JK, Bolinska H, Zaslonka J, Zareba W. Relationship between heart rate turbulence and heart rate, heart rate variability, and number of ventricular premature beats in coronary patients. Journal of cardiovascular electrophysiology 2004;15(7):731-737.

[9] Rojo Alvarez JL, Barquero-Pérez O, Mora-Jiménez I,
Everss E, Rodríguez-González AB, García Alberola A. Heart rate turbulence denoising using support vector machines. IEEE Transactions on Biomedical Engineering 2009;56(2):310-319.

[10] Solem K, Laguna P, Martínez JP, Sörnmo L. Model-based detection of heart rate turbulence. IEEE Transactions on Biomedical Engineering 2008;55(12).

[11] Hastie T, Tibshirani R, Friedman J. The elements of statistical learning: data mining, inference and prediction. 2 edition. Springer, 2009.

[12] Gonçalves P, Risco D, Fernández-Llario P, Barquero-Pérez O, Serrano E, Hermoso-de Mendoza J, Mateos C. A new method for ageing wild boar using dental measures. Ecological Indicators 2016;62:328-332.

[13] James G, Witten D, Hastie T, Tibshirani R. An introduction to statistical learning. Springer, 2013.

[14] González-Carrillo J, García-Alberola A, Saura D, Carrillo P, López R, Sánchez-Muñoz JJ, Martínez J, Valdés M. Impacto de la angioplastia primaria en la indicación de desfibrilador implantable en pacientes con infarto de miocardio. Rev Esp Cardiol 2003;56(12):52-56.

Address for correspondence:

O Barquero-Pérez

Department of Signal Theory and Communications

University Rey Juan Carlos. DIII:D-207, Camino del Molino s/n 28943 - Fuenlabrada (Madrid), Spain

Phone: +3491488 8462

oscar.barquero@urjc.es 